\title{
Ankündigungen der Deutschen Vereinigung für Sportwissenschaft
}

\section{dvs-Sommerakademie 1988}

Zur Förderung des sportwissenschaftlichen Nachwuchses führt die Deutsche Vereinigung für Sportwissenschaft vom 15. bis 25. Juli 1988 in Berlin (Willi-Weyer-Akademie des Deutschen Sportbundes) ihr zweite Sommerakademie mit dem Titel „Sportwissenschaft als rationale Konstruktion sportlicher Wirklichkeit" durch. Die damit zu verbindenden (wissenschaftstheoretischen) Themen werden von namhaften Wissenschaftlern behandelt, z. B. Sportwissenschaft als Institution (Prof. Dr. Digel), Finalisierung (Prof. Dr. Becker), Alltag und (Sport-)Wissenschaft (Prof. Dr. G. Böhme), Verantwortung (Prof. Dr. Spinner), Quantitative Verfahren (Prof. Dr. Kriz), Qualitative Verfahren (Prof. Dr. Oevermann) und Beratung (Priv.-Doz. Dr. Dewe). Die Leitung haben Prof. Dr. P. Becker (Marburg), Prof. Dr. H. Digel (Darmstadt) und G. Drexel (Tübingen). Weitere Informationen sind bei der dvsGeschäftsstelle (Universität Bielefeld, Abteilung Sportwissenschaft, Postfach 8640, 4800 Bielfeld 1) erhältlich.

\section{Jahrestagung der Sektion Sportsoziologie der Deutschen Vereinigung für Sportwissenschaft}

Vom 16.-18. 6. 1988 findet die o.g. Jahrestagung zum Thema „Beratungsleistungen der Sportsoziologie “ in Konstanz statt. Die Möglichkeiten und die Grenzen wissenschaftlicher Beratung, speziell sportsoziologischer, sind die Schwerpunkte dieser Tagung. Referenten: Dr. Bonß (Institut für Sozialforschung Hamburg), Prof. Dr. Heinemann (Universität Hamburg), Dr. Schaible (LSV Baden-Württgb.). Kontakt: G. Anders, Bundesinstitut für Sportwissenschaft, Carl-Diem-Weg 4, 5000 Köln 41.

\section{Literatur-Neuerscheinungen}

Aвraham, Anke: Identitätsprobleme in der Rhythmischen Sportgymnastik. (Beiträge zur Lehre und Forschung im Sport. Bd. 94.) Schorndorf: Hofmann 1986. 179 S.; DM 23,80. Adrian, Marlene J. (Hrsg.): Sports women. (Medicine and sport science. Vol. 24.) Basel usw.: Karger 1987. 157 S.; DM 177,- .

Aldershoff-GAEMERS, Lidy (Bearb.): Sport on television and sport-sponsorship. Hilversum: Intomart 1986. $134 \mathrm{~S} . ;$ hfl 420,-

Allison, Lincoln (Hrsg.): The politics of sport. Manchester: Univ. Pr. 1986. VIII, 264 S.; $£ 25$, -

Andrecs, Hermann / Redl, Sepp (Hrsg.): Leibeserziehung - Enquete '85. Zur Situation der Leibesübungen in den Schulen Österreichs. (Theorie und Praxis der Leibesübungen. Bd. 53.) Wien: Österr. Bundesverl. 1985. 216 S.; DM 18,30. 